\title{
Hacia una propuesta educativa para fomentar la identidad profesional enfermera en estudiantes de pregrado
}

\author{
Rosalba Pulido de Castellanos ${ }^{1}$; Aníbal Alfonso Teherán Valderrama ${ }^{2}$; María Antonina Román Ochoa ${ }^{3}$
}

1 Doctorado en Educación de la Universidad Santo Tomás (Bogotá, Colombia), Directora de la Tesis Doctoral "Propuesta educativa para fomentar la Identidad Profesional Enfermera en estudiantes de pregrado"

rpulido0730@gmail.com

2 Docente Investigador de la Fundación Universitaria Juan N. Corpas (Bogotá, Colombia)

anibal.teheran@juanncorpas.edu.co

3 Candidata a Doctora en Educación de la Universidad Santo Tomás (Bogotá, Colombia), Docente de la Fundación Universitaria Juan N. Corpas antonina.roman@juanncorpas.edu.co

Correspondencia:

Dra. María Antonina Román Ochoa, antonina.roman@juanncorpas.edu.co

Como citar: Pulido R, Teherán AA, Román MA. Hacia una propuesta educativa para fomentar la identidad profesional enfermera en estudiantes de pregrado. Cuarzo 2015; 21 (1): 16-24.

Recibido: 15 de marzo de 2015. Aceptado: 30 de mayo de 2015. Publicado: 30 de junio de 2015.

Licencia creative commons (c) $($ ) $(9)$

\section{Resumen}

OBJETIVO: Determinar factores relacionados con la Identidad Profesional con el fin de proponer una estrategia educativa que contribuya a fomentarla entre los estudiantes de pregrado de Enfermería.

METODOLOGÍA: Se realizó un diseño metodológico mixto, utilizando como variable de resultado el Puntaje de Identidad Profesional Enfermera (IPE) según características sociodemográficas y de aspectos profesionales. A las variables cuantitativas se les calcularon las medidas de tendencia central y dispersión y a las numéricas la proporción; como medida de asociación se utilizó el OR y de precisión el IC $95 \%$.

RESULTADOS: Se obtuvieron 95 encuestas; la mediana de Identidad Profesional Enfermera fue de 93; los mayores puntajes de IPE (100) se encontraron en profesionales que están cursando o han terminado estudios de doctorado. El 93,7 \% de los enfermeros percibió subordinación en los escenarios de práctica, el $87 \%$ no siente que su labor sea apreciada por la sociedad y el $27 \%$ no percibe su práctica profesional como estimulante.

CONCLUSIÓN: Se identificaron posibles factores relacionados con la IPE desde los ámbitos vocacionales y nivel educativo, también se percibieron vacíos en la fundamentación técnico-científica básica y disciplinar en la formación de pregrado. Se debe ejecutar un nuevo proceso de validación de la escala de IPE con el fin de dar validez a los posibles factores predictores identificados en la presente investigación.

PALABRAS CLAVE: Educación en Enfermería, enfermería, rol de la enfermera, autonomía profesional. 


\section{Towards an educational proposal to promote undergraduates' professional identity}

OBJECTIVE: To determine the factors associated with Professional Identity, with the aim of proposing an educational strategy that fosters it among the nursing program's undergraduate students.

METHODOLOGY: A mixed methodological design was followed, using the Nurse Professional Identity's score (IPE) as variable according to socio-demographic characteristics and professional aspects. Quantitative measures had core trend and dispersion measures calculated and numeric variables had proportion calculated; OR was used as association measure and $95 \%$ IC was used as precision measure.

RESULTS: 95 surveys were conducted; the mean for the Nurse Professional Identity was 93; the highest scores (100) came from professionals that are studying or have finished doctorate studies. $93.7 \%$ of the nurses perceived subordination within their practice scenarios; $87 \%$ do not feel his/her task is appreciated by society and $27 \%$ do not perceive his/her professional practice as stimulating.

CONCLUSION: Possible factors related to Nurse Professional Identity were identified from vocational and educational spheres, also, gaps in basic technical-scientific and disciplinary training were identified in the undergraduate studies. A new validation process for the Nurse Professional Identity scale must be executed with the objective of validating possible predicting factors identified in this research.

KEYWORDS: education, nursing, nurse's Role, professional autonomy.

\section{Introducción}

El ejercicio de la enfermería se ha visto enmarcado en situaciones tales como poco reconocimiento por parte de la sociedad, salarios inadecuados, condiciones de trabajo precarias, bajo índice de enfermeros por habitantes, por mencionar algunas $(1,2)$. Las organizaciones gremiales y académicas de enfermería han venido trabajando en afianzar la autonomía profesional para mejorar el liderazgo y reconocimiento de la carrera. Por tanto, resulta de gran importancia fortalecer la Identidad Profesional con miras a lograr un mejor posicionamiento de la profesión.

Se han realizado diversos estudios para determinar los elementos que influyen en la configuración de la Identidad Profesional de los enfermeros, encontrando de importancia la educación continuada $(3,4)$, los escenarios de práctica a los que asisten los estudiantes $(5,6,7)$ y la socialización de aquellos durante el pregrado $(6,8,9,10)$, entre otros. De igual manera, hay reportes que analizan la Identidad Profesional en estudiantes de enfermería (7, 11) e investigaciones que profundizan en la construcción de la misma y su relación con la satisfacción laboral y la intención de continuar ejerciendo la profesión $(12,13)$. Si bien se hace referencia a que la educación es importante en la configuración de la Identidad Profesional, en la literatura revisada no se hallaron estudios que sugieran cómo reforzarla desde la formación de pregrado, hecho que es abordado en la investigación en curso, en el marco del Doctorado en Educación de la Universidad Santo Tomás (Bogotá, Colombia) y cuyo objetivo es el de determinar factores relacionados con la Identidad Profesional con el fin de proponer una estrategia educativa que contribuya a fomentarla entre los estudiantes de pregrado de Enfermería.

La etapa cuantitativa del estudio permitió tener una aproximación de la percepción de enfermeros de diferentes áreas de desempeño y con distintos niveles de formación (desde pregrado hasta doctorado) sobre aspectos que han sido considerados de importancia en la configuración de la Identidad Profesional, así como conocer el comportamiento de los factores identificados por Sharbaugh (12) como determinantes de la Identidad Profesional, elementos que servirán de base para la exploración cualitativa propuesta como segunda etapa de la investigación, para, con base en los hallazgos de las dos etapas, posteriormente proponer una estrategia educativa que contribuya, desde un programa de pregrado, a fortalecer la Identidad Profesional en estudiantes de enfermería.

\section{Metodología}

Tipo de estudio. Diseño cualitativo del orden de la fenomenología, cuantitativo de corte transversal. 
Población objeto de estudio. Profesionales de enfermería ubicados en diferentes regiones de Colombia, en el periodo de abril a mayo de 2014 .

Variables. En la etapa de la investigación que se reporta, se tuvieron en cuenta:

- Variable de resultado primaria: Puntaje de Identidad Profesional según características sociodemográficas y de aspectos profesionales de los enfermeros encuestados.

- Variable de resultado secundaria: Puntaje de Identidad Profesional según percepción de aspectos de la formación profesional de enfermería en Colombia.

- Variables sociodemográficas: Edad estratificada en intervalos de 10 años, partiendo desde 25 o menos años hasta mayor o igual a 66 años; sexo; estado civil; nivel educativo (identificando el máximo estudio o grado realizado); estrato socioeconómico; barrio y ciudad de residencia.

- Variables profesionales: Aspectos vocacionales de la profesión (escogencia de enfermería como primera opción y antecedentes de una carrera técnica previa), estudios de postgrado (culminación de estudios de especialización, maestría o doctorado, influencia de estudios de posgrado en reconocimiento profesional, condición salarial y/o posición laboral) y participación en actividades de educación permanente y continuada.

- Percepción de aspectos formativos en enfermería: Orientación de la carrera hacia aspectos procedimentales o teóricos; enseñanza de la enfermería enfocada en aspectos biomédicos; dependencia de los programas de medicina; percepción de subordinación de los profesionales de enfermería en escenarios de práctica formativa; promoción del desarrollo del liderazgo y del trabajo en equipo, y déficit de los programas de enfermería en la Fundamentación técnico-científica básica o disciplinar.

Plan de recolección de datos. Se consideraron varias escalas de IP, dentro de las que se contaban la Escala de Identidad Profesional MCPIS-9, la Escala de Identidad Profesional de Adams, et al. (14), la Escala de Valores Profesionales del Enfermero de Weis y Shank (15) y la Escala de Identidad Profesional Enfermera de Sheila Sharbaugh (12), escogiendo esta última debido a su dis- ponibilidad y a que las otras se adecuaban generalmente a la población en la etapa de pregrado. El uso de la escala traducida al español fue autorizado por su autora, Sheila Sharbaugh. A partir de la licencia de la autora, se realizó la traducción de los 24 ítems y se envió para revisión por parte de traductor certificado.

Se diseñó un formato tipo encuesta que incluyó las variables previamente mencionadas $\mathrm{y}$, en el tercer punto, se incorporó la escala con las modificaciones sugeridas por el traductor certificado. Posteriormente, se procedió a realizar una prueba piloto con 20 enfermeros colombianos, ubicados en diferentes ámbitos laborales: administrativo en el sector del aseguramiento, administrativo en el área clínica, docente, directivo y administrativo en el sector educativo, asistencial en el área clínica en diferentes servicios y asistencial en el área ambulatoria; y con diferentes niveles de escolaridad: pregrado, especialización clínica, especialización administrativa, maestría y candidato a doctor. Se evaluó la pertinencia de las sugerencias con dos pares evaluadores para decidir nuevos ajustes a la encuesta definitiva y, luego de ello, se aplicó la encuesta a la población objeto de estudio.

Se tomó una muestra por conveniencia de bases de datos públicas donde figuraban nombres y correos electrónicos de profesionales de enfermería, a los que se les envío la encuesta definitiva y se determinó un plazo 60 días como punto de corte para recolectar la muestra requerida e iniciar el proceso de análisis de los resultados.

Plan de análisis. Los datos recolectados se agruparon en tablas y gráficos según su pertinencia; a las variables cuantitativas se les calcularon las medidas de tendencia central y dispersión y a las categóricas el conteo y la proporción; las diferencias se determinaron con una prueba a dos colas y se estableció una $\mathrm{P}<\mathrm{a} 0,05$ como significativa. Por medio de la prueba de Kolmorogov-Smirnov se determinó la normalidad de la distribución de las variables cuantitativas, con el fin de establecer el uso de medidas agrupadas de tipo localización como representativas de los datos; las diferencias entre variables categóricas se establecieron con una prueba de independencia Chi cuadrado.

Se realizó un modelo de regresión lineal tomando como variable dependiente el puntaje de Identidad Profesional (y) y como variable independiente (x) los años de graduación. Se realizó una prueba de consistencia in- 
terna del instrumento de medición de la escala de Identidad Profesional, implementado un punto de corte de $\alpha$ Cronback de 0.7 para establecer lo referido; como medida de precisión se utilizó el IC $95 \%$.

\section{Aspectos éticos}

Según la resolución 008430 de 1993, esta investigación se clasificó Sin Riesgo; se respetó la confidencialidad de los datos y la recolección de la información contó con la aprobación del Comité de Investigaciones de la Universidad Santo Tomás.

\section{Resultados}

\section{Características generales}

Se procesaron 102 encuestas, de las cuales se excluyeron siete por diligenciamiento incompleto de la información solicitada, quedando para el análisis final 95 profesionales en enfermería.

Para el análisis de la variable "Con respecto a la formación profesional de enfermería, usted considera que: La carrera está centrada prioritariamente en...", se excluyó un encuestado por inconsistencia en la información suministrada.

Se rechazó la hipótesis de normalidad en la distribución de los datos de las variables puntaje de Identidad Profesional (IPE) y años de graduado (KolmorogovSmirnov). La mediana (RI) de IPE y de años de graduado fue 93 (86-97) y $16(11,5-25,5)$ respectivamente; con respecto a la IPE no se encontraron diferencias entre las sub-categorías de las variables sociales y demográficas establecidas. La mayoría de los profesionales tenían entre 36 y 45 años de edad (47,4 \%; IC95 \% 37,3 \% - 57,4 \%), eran mujeres (93,7 \%; IC $95 \% 88,8 \%$ - 98,6 \%) y pertenecían al estrato socioeconómico 3 (37,9\%; IC $95 \%$ 28,1 $\%$ - 47,6\%). En la Tabla 1 se presentan estos resultados.

Se identificó que al momento de la graduación al menos se tenía un puntaje de 91 en la escala de IPE, sin encontrar relación entre el puntaje de IPE y los años de graduación (R2 0.002; P: 0,652).

\section{Factores de las etapas de pregrado y postgrado relacionados con IPE}

Se propusieron varios factores que desde el ámbito académico han sido relacionados con cambios en la IPE en el área de enfermería, dentro de ellos se encontró que alrededor de uno de cada 10 profesionales estudió una carrera técnica antes de ingresar a enfermería, uno de cada cinco no escogió enfermería como primera opción de estudio, tres de cada cuatro han culminado un posgrado y la mayoría considera que dichos estudios han influido positivamente en su desempeño laboral y en su reconocimiento profesional. Entre tanto, las medianas de puntaje IPE más altas se identificaron entre los profesionales que no escogieron enfermería como primera opción y no culminaron estudios de posgrado; entre aquellos que culminaron estudios de posgrado pero refirieron que estos no habían sido influyentes en su desempeño laboral o reconocimiento profesional, se identificaron las medianas más bajas de puntaje de IPE, sin encontrar diferencias estadísticas entre los profesionales que respondieron sí o no a cualquiera de las preguntas mencionadas (Gráfica 1A y 1B).

TABLA 1. Características sociales, demográficas y media de puntaje de identidad profesional de profesionales en Enfermería, Colombia, 2014.

\begin{tabular}{|c|c|c|}
\hline $\begin{array}{c}\text { Características } \\
\text { generales }\end{array}$ & $\mathrm{n}$ & $\begin{array}{c}\text { Puntaje identidad } \\
\text { profesional }\end{array}$ \\
\hline Edad (\%) & media (DE) & \\
\hline$\leq 25$ & $6(6,3)$ & $92,3(7)$ \\
\hline 26 a 35 & $14(14,7)$ & $93,3(8)$ \\
\hline 36 a 45 & $45(47,4)$ & $90,5(8)$ \\
\hline 46 a 55 & $17(17,9)$ & $93,7(5)$ \\
\hline 56 a 65 & $11(11,6)$ & $93,1(8)$ \\
\hline$\geq 66$ & $2(2,1)$ & $85,5(12)$ \\
\hline \multicolumn{3}{|l|}{ Sexo } \\
\hline Femenino & $89(93,7)$ & $92(7)$ \\
\hline Masculino & $6(6,3)$ & $89,5(7)$ \\
\hline \multicolumn{3}{|l|}{ Estado civil } \\
\hline Casado (a) & $45(47,4)$ & $91,4(7)$ \\
\hline Soltero (a) & $23(24,2)$ & $92,1(9)$ \\
\hline Divorciado & $13(13,7)$ & $93(7)$ \\
\hline Separado & $6(6,3)$ & $91,7(9)$ \\
\hline Unión Libre & $8(8,4)$ & $92(8)$ \\
\hline \multicolumn{3}{|c|}{ Estrato socioeconómico } \\
\hline 2 & $18(18,9)$ & $92,2(9)$ \\
\hline 3 & $36(37,9)$ & $91,8(7)$ \\
\hline 4 & $14(14,7)$ & $95(6)$ \\
\hline 5 & $22(23,2)$ & $89,6(7)$ \\
\hline 6 & $5(5,3)$ & $91(9)$ \\
\hline
\end{tabular}

No se encontraron diferencias $(\mathrm{p}>0,05)$ entre los subgrupos de cada categoría. 
Con relación a la frecuencia de estudios de posgrado, se identificó que la mitad ha realizado estudios de especialización, uno de cada cuatro maestría y menos del $5 \%$ estudios de doctorado, entre estos últimos se identificó el subgrupo con mayor puntaje en la escala de IPE (Gráfica 2).

Con respecto a la opinión sobre aspectos fundamentales de los procesos formativos durante la carrera, la mayoría de los profesionales refirió que la carrera no está centrada en aspectos teóricos o enfocada en conceptos biomédicos; percibe subordinación de los profesionales en escenarios de práctica formativa; identifica vacíos en la fundamentación técnico-científica básica y disciplinar; refiere que se les promueve hacia el desarrollo del liderazgo y del trabajo en grupo y que se incentiva el conocimiento de la legislación en el contexto del ejercicio de la profesión (Tabla 2).

Al dicotomizar el puntaje de IPE implementando como punto de corte 100 , no se identificó relación alguna entre aquellos con buena identidad profesional $(\geq 100)$ y variables demográficas o sociales como sexo, grupos de edad, estado civil, estrato socioeconómico y ciudad de residencia.

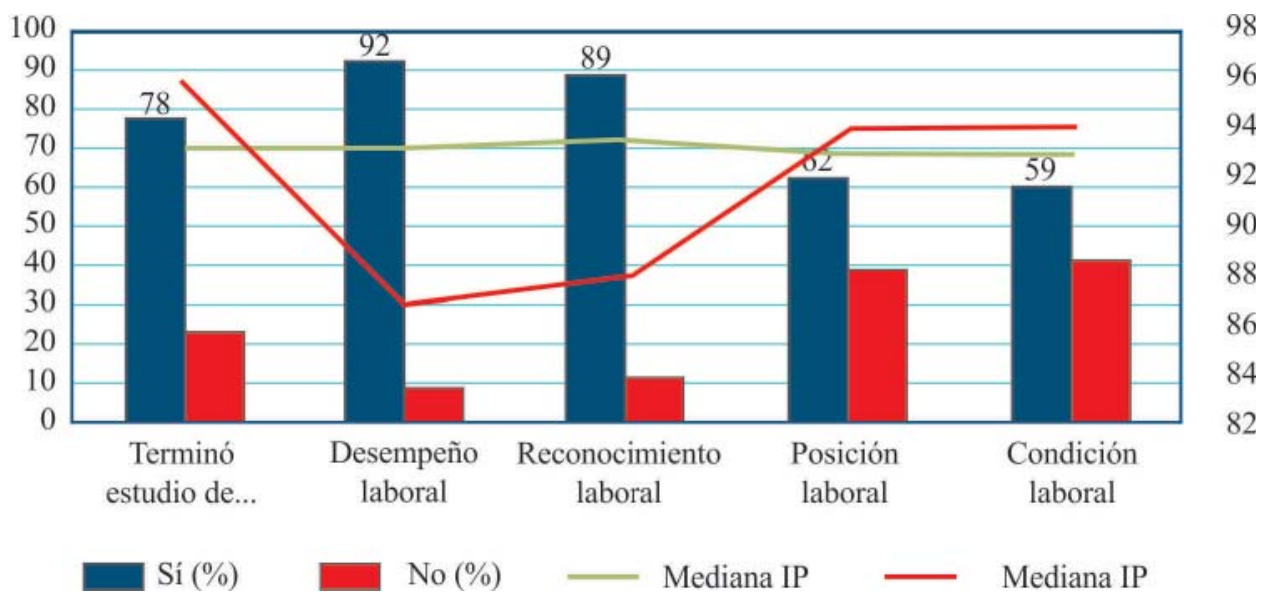

GRÁFICA 1A. Mediana de puntaje de identidad profesional (IP) y estudios de posgrado e influencia en aspectos laborales, Colombia, 2014.

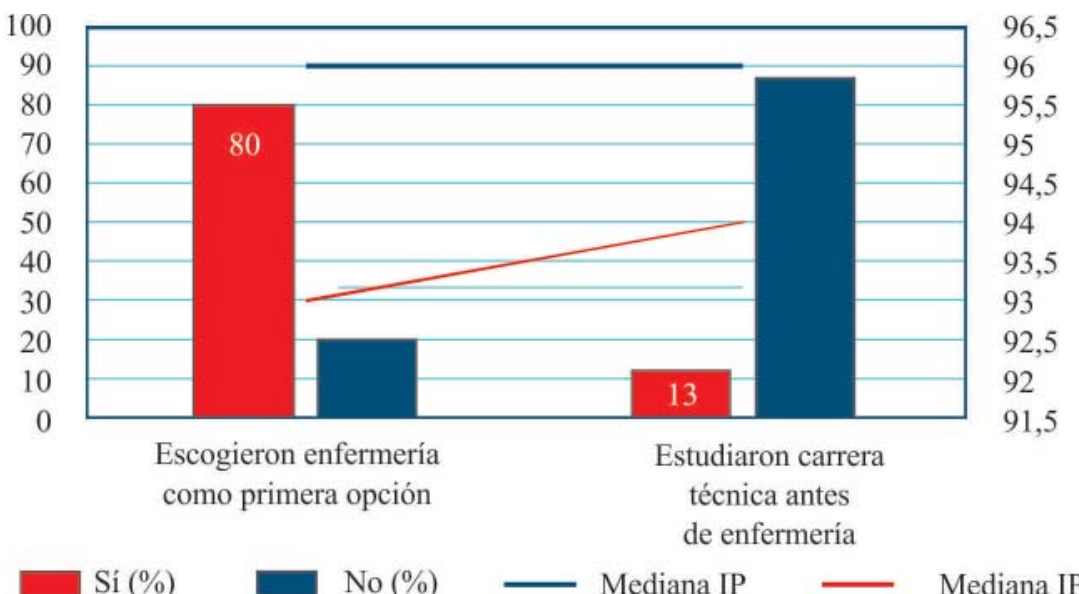

GRÁFICA 1B. Mediana de puntaje de identidad profesional (IP) y aspectos de vocación, Colombia, 2014 


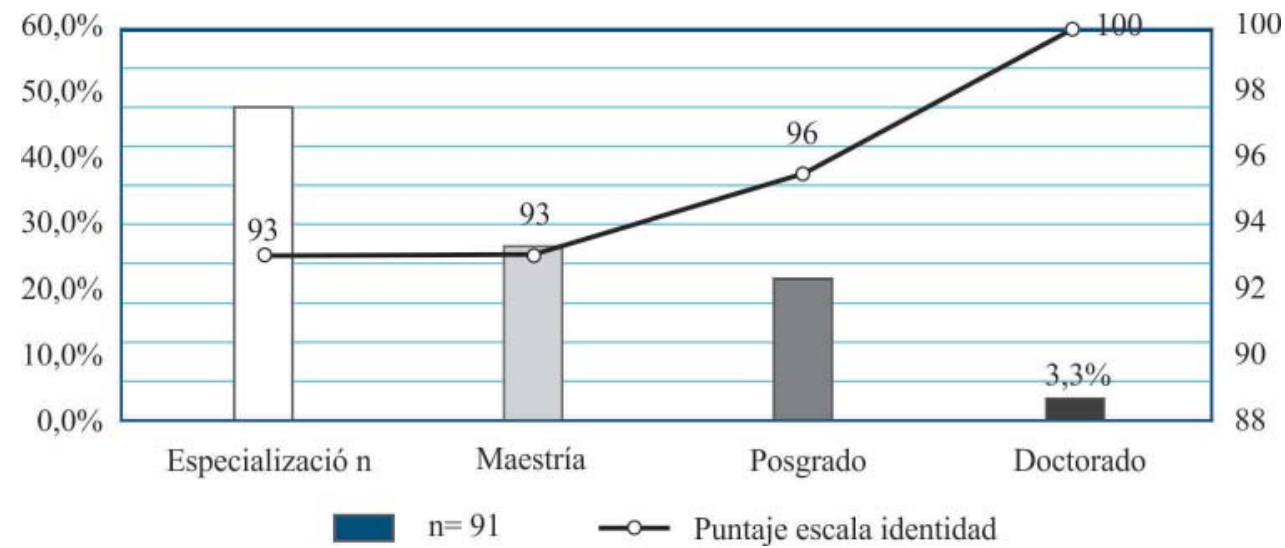

GRÁFICA 2. Frecuencia de nivel académico máximo alcanzado y mediana de puntaje de identidad profesional (IPE), Colombia, 2014. Para este análisis se excluyeron los profesionales que no respondieron esta pregunta en la encuesta

TABLA 2. Evaluación de la percepción de los procesos formativos en Enfermería, Colombia, 2014.

\section{Procesos formativos en la carrera de Enfermería}

\section{Likert, n (\%)

$\leq 2$

\section{Carrera centrada en ...}

Aspectos procedimentales

$49(55)$

$40(45)$

Aspectos teóricos**

$54(60,6)$

$35(39,4)$

\section{Enseñanza enfocada en ...}

Conceptos biomédicos*

$53(59,5)$

$36(40,5)$

Dependencia de los programas de Medicina

$42(47,2)$

$47(52,8)$

Percepción de subordinación de los profesionales en escenarios de práctica formativa***

$11(12,4)$

$78(87,6)$

\section{Vacíos en...}

Fundamentación técnico - científica básica***

Fundamentación disciplinar**

Promoción de ...

Desarrollo de liderazgo**

$\begin{array}{ll}35(39,4) & 54(60,7) \\ 16(17,9) & 73(82,1)\end{array}$

Trabajo en grupo***

\section{Se incentiva ...}

Conocimiento de la legislación en el contexto del ejercicio de la profesión***

$30(33,7)$

$*: \mathrm{P}<0,05 ; * *: \mathrm{P}<0,01 ; * * *: 0,001$. Prueba a dos colas. 
Se identificó una posible relación entre los profesionales que respondieron que la enseñanza en Enfermería está enfocada en conceptos biomédicos y aquellos con buen puntaje de IPE (P: 0,007; dato no mostrado), es necesario tener en cuenta que fue muy baja la frecuencia de profesionales que respondieron a esta pregunta, generando así limitaciones en la aplicabilidad de la misma. Al realizar la misma prueba de correlación entre el puntaje de IPE y el puntaje obtenido en las preguntas que trataban sobre los procesos formativos durante el transcurso de la carrera, no se identificó correlación alguna $(\mathrm{P}>0,05)$, descartando así el hallazgo previamente mencionado.

\section{Evaluación de la escala de Identidad Profesional (IPE)}

Se realizó una prueba de consistencia interna a la escala traducida de Identidad Profesional (IPE) y se calculó un $\alpha$-Cronbach de 0,532 , estableciendo limitaciones metodológicas para la aplicación de los resultados. A pesar de este hallazgo se analizaron las respuestas a las preguntas establecidas en la escala.

Se encontró que el $100 \%$ de los enfermeros participantes manifestó total acuerdo con aspectos que resultan de vital importancia en la configuración de la Identidad Profesional, tales como sentir que la enfermería forma parte de su vida, promover los aspectos positivos de esta, considerar de importancia reflexionar sobre su práctica profesional, considerar la profesión como un estímulo intelectual y estimar su profesión como algo muy valioso.

Aunque no todos los participantes manifestaron estar en total acuerdo, un alto porcentaje lo estuvo con aspectos que en la literatura sobre Identidad Profesional son considerados como determinantes en la configuración de la Identidad Profesional Enfermera. El 98 \% se considera competente y el $95 \%$ tiene altos estándares en su práctica profesional. E1 $95 \%$ de los enfermeros encuestados respondió que la práctica de la enfermería le satisface totalmente y el $98 \%$ siente armonía con los pacientes a quienes cuida o con las personas con las que interactúa, en el caso de cargos administrativos.

Llama la atención que el $27 \%$ de los participantes haya respondido que no siente estimulante su práctica profesional. De igual manera, resalta que el $51 \%$ no se sienta en sintonía con otros enfermeros, respuesta que no se esperaría teniendo en cuenta que el $87 \%$ del mismo grupo contestó que se siente respetado por sus colegas.
De otra parte, en consonancia con la preocupación de las asociaciones y agremiaciones académicas y profesionales, tanto en Colombia como en Latinoamérica y el mundo, se encuentra baja participación de los profesionales en espacios de discusión gremial o de participación interprofesional, $56 \%$ de los encuestados no pertenece a ninguna asociación profesional o académica y $25 \%$ no está involucrado en comités de práctica profesional en su sitio de trabajo.

Finalmente, se evidencian dos aspectos que con frecuencia se analizan y discuten en la literatura y en foros y congresos profesionales de enfermería: el $33 \%$ manifestó no sentirse independiente en su ejercicio profesional y el $87 \%$ considera que la labor de enfermería no es apreciada por la sociedad.

\section{Discusión}

En esta etapa de la investigación no se identificaron factores relacionados con la Identidad Profesional en el punto de corte establecido por la escala implementada. Algunas variables de tipo vocacional o referentes al nivel académico presentaron una tendencia positiva en relación con la Identidad Profesional.

El tema de la Identidad Profesional Enfermera parece no tener aún una mayor relevancia en nuestro país. Hay poca disponibilidad de literatura relacionada con estudios o análisis sobre la Identidad Profesional Enfermera en Colombia y, al revisar la información disponible en los sitios web de los diferentes programas de enfermería, no se hacen evidentes estrategias o contenidos enfocados al fortalecimiento de la Identidad Profesional de los estudiantes de enfermería.

Aspectos como contrataciones temporales y a través de intermediarios, salarios inadecuados y condiciones inapropiadas de trabajo, que afectan a un alto número de profesionales de enfermería en Colombia (2), son factores determinantes en su ejercicio profesional y pueden estar afectando la Identidad Profesional Enfermera.

En la investigación se encuentra que al estratificar por sexo o por el antecedente de estudios de una carrera técnica en el área de la salud previa a la de enfermería, no se identificaron diferencias entre los subgrupos con alta o baja Identidad Profesional, a diferencia de los hallazgos de Worthington et al. (11), en donde se encontró una relación positiva en el grupo de las mujeres y aquellos 
con una carrera técnica previa; se debe aclarar que dicho estudio fue realizado entre estudiantes de pregrado de enfermería, utilizando una escala diferente a la Escala de Sharbaugh (12). Ello da indicios de que se requeriría realizar un trabajo de indagación complementario, para establecer el efecto de los instrumentos aplicados en cada una de las investigaciones reseñadas dado el hecho de que en un caso se trata de estudiantes de pregrado de enfermería y en el otro de enfermeros profesionales.

El punto anterior se refuerza al tener en cuenta que Cowin et al. (7), encontró una disminución en los niveles de Identidad Profesional, evaluada mediante 5 pruebas psicométricas, al comparar los estudiantes de primer año con los de tercer año; por su parte, Coster et al. (16), refiere que en las disciplinas de salud que participaron en su estudio de educación interprofesional (incluida la enfermería), hubo una fuerte Identidad Profesional al entrar a la universidad, la cual disminuyó con el tiempo.

El hecho de que el grupo de enfermeros que está estudiando o ha culminado estudios de doctorado haya tenido la media de Identidad Profesional más alta es un hallazgo coincidente con la literatura en lo relacionado con la influencia positiva de la educación continuada en la Identidad Profesional. Al respecto, Franco \& Tavares (3), utilizando una escala diseñada por ellos, encontraron una relación directamente proporcional entre la percepción de Identidad Profesional y el adecuado uso de las competencias adquiridas en los procesos de educación continuada. Uno de los hallazgos de Deppoliti (4) tras realizar un estudio cualitativo, fue el hecho de que la educación permanente juega un papel fundamental en la construcción de la Identidad Profesional en enfermeras graduadas. De otra parte, este hallazgo puede ser de relevancia desde el punto de vista de la formación de los futuros enfermeros si se tiene en cuenta la importancia que los estudiantes confieren al "role model" (8), en este caso, un profesor con estudios de doctorado.

La apreciación de subordinación de los enfermeros en los escenarios de práctica, la consideración de pobre autonomía profesional y de muy pobre reconocimiento social, son aspectos que deben ser abordados desde los currículos de enfermería, procurando llenar los vacíos percibidos en aspectos tan importantes como la fundamentación técnico - científica básica y la fundamentación disciplinar.
En el mismo sentido, si bien una tercera parte de los encuestados consideró que en el pregrado se promueve el conocimiento de la legislación en el contexto del ejercicio de la profesión, la escasa vinculación a asociaciones profesionales o académicas evidenciada en las respuestas, sugiere la necesidad de reforzar la formación en democracia y participación ciudadana, con el fin de dar a los futuros profesionales herramientas que contribuyan a analizar de manera crítica aspectos relacionados con el ejercicio de su profesión y motiven la participación en todos aquellos espacios que puedan significar mejoramiento y posicionamiento de su disciplina, propendiendo de esta manera, por el empoderamiento de las futuras generaciones de enfermeros, con miras a reforzar su Identidad Profesional.

En la presente investigación se presentaron limitaciones metodológicas dadas por el proceso de validación de la escala de Identidad Profesional Enfermera; sin embargo, el puntaje de consistencia interna posterior al proceso de traducción y de prueba piloto no fue distante al de la escala original. Cabe mencionar que hasta el momento no se han encontrado reportes de investigación que evalúen la Identidad Profesional Enfermera en Colombia utilizando alguna de las escalas previamente mencionadas.

De otra parte, es necesario referir que el carácter descriptivo del diseño metodológico, aunado al muestreo por conveniencia, limita parcialmente la inferencia de los resultados; no obstante, posterior a la obtención de los resultados cuantitativos descritos, la presente investigación será complementada con el componente cualitativo que permitirá profundizar los hallazgos evidenciados con el fin de proponer una estrategia educativa que fortalezca la Identidad Profesional Enfermera en estudiantes de pregrado. De igual manera, el subgrupo de preguntas referentes a la percepción de la formación en el pregrado de enfermería debe someterse a un proceso de validación interna.

Como conclusión, identificamos una posible relación entre la Identidad Profesional Enfermera y variables tales como no haber escogido enfermería como primera opción o tener estudios de doctorado; no identificamos relación alguna con variables de tipo demográficas o sociales. Los enfermeros encuestados percibieron que los programas de enfermería muestran dependencia de los programas de medicina; subordinación de los enfermeros en los escenarios de las prácticas formativas y vacíos en la formación en lo referente a la fundamentación técnico-científica básica y a la fundamentación disciplinar. 
Estos resultados preliminares deben ser complementados con los resultados de la fase cualitativa del estudio, para así poder generar una propuesta educativa con miras a fortalecer la configuración de la Identidad Profesional de los estudiantes de enfermería. De igual manera, resulta necesaria la realización de estudios de investigación en este mismo sentido y que permitan hacer seguimiento al impacto de estrategias educativas tendientes a fortalecer la Identidad Profesional.

Se deben optimizar los procedimientos encaminados a mejorar la consistencia interna de la escala posterior al proceso de traducción al idioma español y considerar la influencia, en el punto de corte de la escala, de las diferencias culturales y de ejercicio profesional en el contexto colombiano en relación con el país de origen de la escala (Estados Unidos) antes de emitir juicios de valor sobre los resultados de la aplicación de la escala y factores de riesgo asociados con la Identidad Profesional Enfermera.

\section{Aclaraciones}

El presente trabajo corresponde a la presentación de resultados parciales de la Tesis Doctoral "La identidad profesional en la formación de pregrado en enfermería" - Doctorado en Educación Universidad Santo Tomás. Bogotá, Colombia.

CONFLICTO DE INTERESES: los autores declaran no tener conflicto de intereses.

\section{Referencias}

1. Organización Mundial de la Salud - OMS. Informe sobre la salud en el mundo 2006 - Colaboremos por la salud [libro en internet]. Ginebra: Organización Mundial de la Salud; 2006 [consultado 26 de abril de 2013]. Disponible en: http:/www.who.int/whr/2006/ es/

2. ANEC (2014). Base de datos del RUN aporta indicadores sociolaborales de profesionales de enfermería. Publicación oficial de la Asociación Nacional de Enfermeras de Colombia 2014; (78): 44-45.

3. Franco M, Tavares P. The influence of professional identity on the process of nurses' training: an empirical study. Leadersh Health Serv. $2013 ; 26(2):$ 118-34.
4. Deppoliti D. Exploring how registered nurses construct professional identity in hospital settings. J Contin Educ Nurs. 2008; 39(6): 255-62.

5. Cárdenas L. Construcción de la identidad de enfermería: una visión bajo el curriculum oculto. Rev. urug. Enferm. 2007; 1(2): 13-19.

6. Serra M. Learning to be a Nurse. Professional Identity in Nursing Students. Sisifo. Educational Sciences Journal. 2008; (5): 65-76.

7. Cowin LS, Johnson M, Wilson I, Borgese K. The psychometric properties of five Professional Identity measures in a sample of nursing students. Nurse Educ Today. 2013; 33(6); 608-13.

8. duToit D. A sociological analysis of the extent and influence of professional socialization on the development of a nursing identity among nursing students at two universities in Brisbane, Australia. J Adv Nurs. 1995; 21, 164-71.

9. Cook T, Gilmer M, Bess C. Beginning students' definitions of nursing: an inductive framework of professional identity. J Nurs Educ. 2003; 42(7), 311-17.

10. Pratt M, Rockmann KW, Kaufmann J. (2006). Constructing Professional Identity: The Role of Work and Identity Learning Cycles in the Customization of Identity among Medical Residents. Acad Manage J. [revista en internet] 2006. [consultado 14 abr 2014]; 49(2): 235-62. Disponible en http://www.jstor.org/stable/20159762

11. Worthington M, Salamonson Y, Weaver R, Cleary M. Predictive validity of the Macleod Clark Professional Identity Scale for undergraduate nursing students. Nurse Educ Today. 2013; 33 : 187-91.

12. Sharbaugh S. Relationships among nurses' professional identity, career satisfaction, occupational commitment, and intent to stay [Tesis Doctoral]. Chester: Widener University - School of Nursing; 2009.

13. Gregg S. Professional identity of japanese nurses: Bonding into Nursing [Tesis Doctoral]. Colorado: University of Colorado School of Nursing; 2000.

14. Adams K, Hean S, Sturgis P, Macleod Clark J. Investigating the factors influencing professional identity of first-year health and social care students. Learning in Health and Social Care. 2006; 5(2): 55-68

15. Weis D, Schank M. Development and Psychometric Evaluation of the Nurses Professional Values Scale-Revised. J Nurs Meas. 2009; 17(3): 221-31.

16. Coster S, Norman I, Murrells T, Kitchen S, Meerabeau E, Sooboodoo $\mathrm{E}$ et al. Interprofessional attitudes amongst undergraduate students in the health professions: a longitudinal questionnaire survey. Int J Nurs Stud. 2008; 45: 1667-81. 